\section{MEXAHИKA \\ УДК 532.516:539.3}

\begin{abstract}
Ю. А. Блинков ${ }^{1}$, А. В. Месянжин ${ }^{2}$, Л. И. Могилевич ${ }^{3}$
${ }^{1}$ Блинков Юрий Анатольевич, доктор фризико-математических наук, заведующий кафредрой математического и компьютерного моделирования, Саратовский национальный исследовательский государственный университет им. Н. Г. Чернышевского, BlinkovUA@info.sgu.ru

${ }^{2}$ Месянжин Артем Вячеславович, ведущий математик, ОАО Конструкторское бюро промышленной автоматики, Саратов, a.v.mesyanzhin@gmail.com

${ }^{3}$ Могилевич Лев Ильич, доктор технических наук, просрессор кафедры высшей и прикладной математики, Московский государственный университет путей сообщения императора Николая II (Поволжский срилиал), Саратов, mogilevich@sgu.ru

В современной волновой динамике одним из важных направлений является изучение поведения волн десормаций в упругих оболочках. Известны математические модели волновых движений в бесконечно длинных геометрически нелинейных оболочках, содержащих вязкую несжимаемую жидкость, на базе связанных задач гидроупругости, описываемых уравнениями динамики оболочек и вязкой несжимаемой жидкости, в виде обобщенных уравнений Кортевега де Вриза. Также методом возмущений по малому параметру задачи получены математические модели волнового процесса в бесконечно длинных геометрически нелинейных соосных цилиндрических упругих оболочках, отличающиеся от известных учетом наличия несжимаемой вязкой жидкости между оболочками, на основе связанных задач гидроупругости, которые описываются уравнениями динамики оболочек и несжимаемой вязкой жидкости с соответствующими краевыми условиями, в виде системы обобщенных уравнений КдВ. В представленной статье проведено исследование модели волновых явлений двух геометрически нелинейных упругих соосных цилиндрических оболочек типа Кирхгосра - Лява, содержащих вязкую несжимаемую жидкость, как между ними, так и внутри. Для рассмотренных систем уравнений с учетом влияния жидкости с помощью построения базиса Грёбнера получены разностные схемы типа Кранка-Николсона. Для генерации этих разностных схем использованы базовые интегральные разностные соотношения, которые аппроксимируют исходную систему уравнений. Применение техники базисов Грёбнера позволяет генерировать схемы, для которых с помощью эквивалентных преобразований можно получить дискретные аналоги законов сохранения исходных дифрференциальных уравнений. На основе разработанного вычислительного алгоритма создан комплекс программ, позволяющий построить грасрики и получить численные решения задач Коши при точных решениях системы уравнений динамики соосных оболочек в качестве начального условия.
\end{abstract}

Ключевые слова: нелинейные волны, вязкая несжимаемая жидкость, цилиндрические упругие оболочки.

DOI: $10.18500 / 1816-9791-2016-16-2-184-197$ 


\section{1. ПОСТАНОВКА ЗАДАЧИ}

В современной волновой динамике одним из важных направлений является изучение поведения волн деформаций в упругих оболочках. Для абсолютно жесткой трубы с круговым сечением ламинарное движение вязкой несжимаемой жидкости под действием гармонического по времени перепада давления исследовано в [1]. Для трубы - упругой цилиндрической оболочки - проведено аналогичное исследование в [2-5].

Известны математические модели волновых движений в бесконечно длинных геометрически нелинейных оболочках [6], содержащих вязкую несжимаемую жидкость, на базе связанных задач гидроупругости, описываемых уравнениями динамики оболочек и вязкой несжимаемой жидкости, в виде обобщенных уравнений Кортевега де Вриза (КдВ). Выявлены эффекты влияния вязкой несжимаемой жидкости на поведение волны деформации в оболочке в зависимости от коэффициента Пуассона материала оболочки. В частности, при наличии жидкости в оболочке из неорганических материалов (различные трубопроводы в технологических сооружениях) выявлен экспоненциальный рост амплитуды волны. В случае органического материала (кровеносные сосуды) наличие жидкости приводит к быстрому затуханию волны.

Методом возмущений по малому параметру задачи получены математические модели волнового процесса в бесконечно длинных геометрически нелинейных соосных цилиндрических упругих оболочках [7], отличающиеся от известных учетом наличия несжимаемой вязкой жидкости между оболочками, на основе связанных задач гидроупругости, которые описываются уравнениями динамики оболочек и несжимаемой вязкой жидкости с соответствующими краевыми условиями, в виде системы обобщенных уравнений КдВ. Выявлены эффекты влияния несжимаемой вязкой жидкости между оболочками на поведение волны деформаций в соосных оболочках. Наличие волны деформаций во внешней оболочке приводит к возникновению волны деформаций во внутренней оболочке, которой не было в начальный момент времени, и происходит «перекачка энергии» (через слой жидкости) от внешней оболочки к внутренней, которая сопровождается немонотонным падением амплитуды волны во внешней оболочке и, как следствие, немонотонным снижением скорости её распространения. При этом во внутренней оболочке происходит немонотонное увеличение амплитуды. Вследствие колебаний амплитуд и скоростей с течением времени их скорости и амплитуды выравниваются.

Рассмотрим две соосные бесконечно длинные упругие оболочки на рис. 1, внутри которых находится вязкая несжимаемая жидкость. Введем следующие обозначения: $\delta$ - ширина щели, занимаемой жидкостью, $R$ - радиус срединной поверхности оболочки; $R_{1}=R^{(1)}-\frac{h_{0}^{(1)}}{2}-$ внутренний радиус внешней оболочки; $R_{2}=R^{(2)}+\frac{h_{0}^{(2)}}{2}-$ внешний радиус внутренней оболочки; $R_{3}=R^{(2)}-\frac{h_{0}^{(2)}}{2}-$ внутренний радиус внутренней оболочки, $R^{(1)}, R^{(2)}$ - радиусы срединных поверхностей внешней и внутренней оболочек; $h_{0}^{(1)}, h_{0}^{(2)}-$ их толщины; $p-$ давление в жидкости; $\rho$ - плотность жидкости; $\nu-$ кинематический коэффициент вязкости. Все механические перемещения внутренней оболочки обозначены индексом (2) сверху, а внешней - индексом (1).

Записывая уравнение движения элемента цилиндрической оболочки в перемещениях для модели Киргофа-Лява, считаем материал упругим с линейной зависимостью интенсивности напряжений $\sigma_{i}$ от интенсивности деформаций $e_{i}$ :

$$
\sigma_{i}=E e_{i}
$$

где $E-$ модуль Юнга.

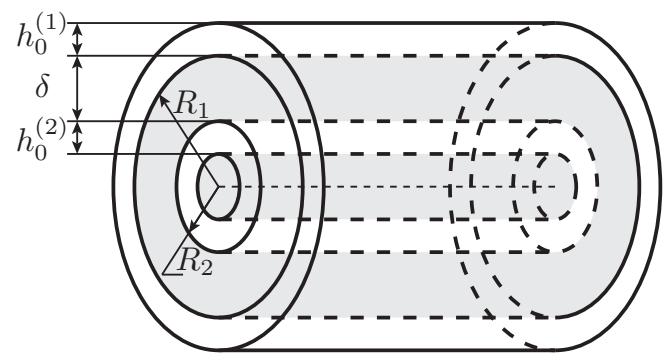

Рис. 1. Упругие бесконечно длинные соосные цилиндрические оболочки

Уравнение движения несжимаемой вязкой жидкости и уравнение неразрывности в цилиндрической системе координат $(r, \Theta, x)$ в случае осесимметричного течения [8,9] записываются в виде

$$
\begin{gathered}
\frac{\partial V_{r}}{\partial t}+V_{r} \frac{\partial V_{r}}{\partial r}+V_{x} \frac{\partial V_{r}}{\partial x}+\frac{1}{\rho} \frac{\partial p}{\partial r}=\nu\left(\frac{\partial^{2} V_{r}}{\partial r^{2}}+\frac{1}{r} \frac{\partial V_{r}}{\partial r}+\frac{\partial^{2} V_{r}}{\partial x^{2}}-\frac{V_{r}}{r^{2}}\right), \\
\frac{\partial V_{x}}{\partial t}+V_{r} \frac{\partial V_{x}}{\partial r}+V_{x} \frac{\partial V_{x}}{\partial x}+\frac{1}{\rho} \frac{\partial p}{\partial x}=\nu\left(\frac{\partial^{2} V_{x}}{\partial r^{2}}+\frac{1}{r} \frac{\partial V_{x}}{\partial r}+\frac{\partial^{2} V_{x}}{\partial x^{2}}\right), \\
\frac{\partial V_{r}}{\partial r}+\frac{V_{r}}{r}+\frac{\partial V_{x}}{\partial x}=0 .
\end{gathered}
$$


На границе оболочек и жидкости на рис. 1 при $r=R_{i}-W^{(i)}$ выполняются условия прилипания жидкости [9]

$$
\begin{gathered}
\frac{\partial U^{(i)}}{\partial t}=V_{x}+U^{(i)} \frac{\partial V_{x}}{\partial x}-W^{(i)} \frac{\partial V_{x}}{\partial r}, \\
-\frac{\partial W^{(i)}}{\partial t}=V_{r}+U^{(i)} \frac{\partial V_{r}}{\partial x}-W^{(i)} \frac{\partial V_{r}}{\partial r} .
\end{gathered}
$$

Здесь $U^{(i)}$ - продольное упругое перемещение оболочки по оси $x ; W^{(i)}-$ прогиб оболочки, положительный к центру кривизны; $t$ - время; $V_{x}, V_{r}$ - проекции на оси цилиндрической системы координат вектора скорости; $x, r-$ цилиндрические координаты.

Уравнения динамики оболочки записываются в виде $[10,11]$

$$
\begin{gathered}
\frac{E h_{0}^{(i)}}{1-\mu_{0}^{2}}\left[U_{x}^{(i)}+\frac{1}{2} U_{x}^{(i)^{2}}+\frac{1}{2} W_{x}^{(i)^{2}}+\frac{h_{0}^{(i)^{2}}}{24} W_{x x}^{(i)}{ }^{2}-\mu_{0} \frac{W^{(i)}}{R^{(i)}}\right]_{x}-\rho_{0} h_{0}^{(i)} U_{t t}^{(i)}=-q_{x}^{(i)}-\tilde{q}_{x}(i-1), \\
\frac{E h_{0}^{(i)}}{1-\mu_{0}^{2}}\left\langle\frac{{h_{0}^{(i)^{2}}}_{12}\left(W_{x x}^{(i)}+U_{x}^{(i)} W_{x x}^{(i)}\right)_{x x}-}{-}\right. \\
-\left\{W_{x}^{(i)}\left(U_{x}^{(i)}+\frac{1}{2} U_{x}^{(i)^{2}}+\frac{1}{2} W_{x}^{(i)^{2}}+\frac{h_{0}^{(i)^{2}}}{24} W_{x x}^{(i)}-\mu_{0} \frac{W^{(i)}}{R^{(i)}}\right)\right\}_{x}- \\
\left.-\frac{1}{R}\left(\mu_{0} U_{x}^{(i)}+\frac{1}{2} \mu_{0} U_{x}^{(i)^{2}}+\frac{1}{2} \mu_{0} W_{x}^{(i)^{2}}+\frac{h_{0}^{(i)^{2}}}{24} \mu_{0} W_{x x}^{(i)}-\frac{W^{(i)}}{R}\right)\right\rangle+ \\
+\rho_{0} h_{0}^{(i)} W_{t t}^{(i)}=(-1)^{i-1} q_{n}+\tilde{q}_{n}(i-1) .
\end{gathered}
$$

Здесь $h_{0}^{(i)}-$ толщины оболочек, $\mu_{0}-$ коэффициент Пуассона, $\rho_{0}-$ плотность; $U^{(i)}, W^{(i)}-$ продольное перемещение и прогиб, положительный к центру кривизны, $x-$ продольная координата, $t-$ время, $q_{x}^{i}, q_{n}$ - напряжения со стороны жидкости, которая находится между оболочками, $\tilde{q}_{x}, \tilde{q}_{n}-$ напряжения со стороны жидкости, которая находится во внутренней оболочке. Нижние индексы у перемещений обозначают соответствующие частные производные.

Напряжения со стороны слоя жидкости определяются формулами

$$
\begin{gathered}
q_{n}=\left.\left[P_{r r} \cos \left(-\widehat{-\bar{n}^{(i)}, \bar{n}_{r}}\right)+P_{r x} \cos \left(\widehat{-\bar{n}^{(i)}}, \bar{i}\right)\right]\right|_{r=R_{i}-W^{(i)}}, \\
q_{x}=\left.\left[P_{r x} \cos \left(-\widehat{-\bar{n}^{(i)}, \bar{n}_{r}}\right)+P_{x x} \cos \left(\widehat{-\bar{n}^{(i)}, \bar{i}}\right)\right]\right|_{r=R_{i}-W^{(i)}}, \\
P_{r r}=-p+2 \rho \nu \frac{\partial V_{r}}{\partial r}, \quad P_{r x}=\rho \nu\left(\frac{\partial V_{x}}{\partial r}+\frac{\partial V_{r}}{\partial x}\right), \quad P_{x x}=-p+2 \rho \nu \frac{\partial V_{x}}{\partial x} .
\end{gathered}
$$

В подходе Эйлера здесь имеем:

$$
\begin{gathered}
\cos \left(-\widehat{\bar{n}}^{(i)}, \bar{n}_{r}\right)=\frac{R_{i}-W^{(i)}}{|\bar{N}|}, \quad \cos \left(\widehat{-\bar{n}^{(i)}, \bar{i}}\right)=-\frac{R_{i}-W^{(i)}}{|\bar{N}|} \frac{\partial W^{(i)}}{\partial x}, \\
\cos \left(\widehat{-\bar{n}, \bar{n}_{r}}\right)=\frac{1}{\left(1+\left(\frac{\partial W^{(i)}}{\partial x}\right)^{2}\right)^{\frac{1}{2}}}, \quad \cos (\widehat{-\bar{n}, \bar{i}})=-\frac{\frac{\partial W^{(i)}}{\partial x}}{\left(1+\left(\frac{\partial W^{(i)}}{\partial x}\right)^{2}\right)^{\frac{1}{2}}}, \\
|\bar{N}|=\left(R_{i}-W^{(i)}\right)\left(1+\left(\frac{\partial W^{(i)}}{\partial x}\right)^{2}\right)^{1 / 2} .
\end{gathered}
$$

Здесь $\bar{n}$ - нормаль к срединной поверхности $i$-й оболочки, $\bar{n}_{r}, \bar{i}-$ орты базиса $(r, \Theta, x)$ цилиндрической системы координат, центр которой расположен на геометрической оси. Если снести напряжения на невозмущенную поверхность оболочки, то можно считать $-\bar{n}=\bar{n}_{r}$ и $\cos \left(\widehat{-}_{\bar{n}, \bar{n}_{r}}\right)=1$, $\cos (\widehat{-\bar{n}, \bar{i}})=0$. Напряжения $\tilde{q}_{x}, \tilde{q}_{n}$ со стороны жидкости, которая находится во внутренней оболочке определяется теми же формулами (5), (6), в которых $\tilde{\rho}-$ плотность жидкости, $\tilde{\nu}-$ коэффициент кинематической вязкости. 


\section{2. ВЫВОД УРАВНЕНИЯ ДИНАМИКИ С УЧЕТОМ НАЛИЧИЯ ЖИДКОСТИ МЕЖДУ УПРУГИМИ ОБОЛОЧКАМИ И ВО ВНУТРЕННЕЙ ОБОЛОЧКЕ}

Принимая длину волны $l$ за характерный размер и обозначая амплитуду продольного перемещения $u_{m}$ и прогиба $w_{m}$, переходим к безразмерным переменным:

$$
W^{(i)}=w_{m} u_{3}^{(i)}, \quad U^{(i)}=u_{m} u_{1}^{(i)}, \quad t^{*}=\frac{c_{0}}{l} t, \quad x^{*}=\frac{x}{l}, \quad c_{0}=\sqrt{\frac{E}{\rho_{0}\left(1-\mu_{0}^{2}\right)}} .
$$

Здесь $c_{0}-$ скорость звука в оболочке. Полагаем

$$
\frac{u_{m}}{l}=\varepsilon=o(1), \quad \frac{R^{(i)}}{l}=O\left(\varepsilon^{1 / 2}\right), \quad \frac{h_{0}^{(i)}}{R^{(i)}}=O(\varepsilon), \quad \frac{w_{m}}{R}=O(\varepsilon) .
$$

Введем полухарактеристические (бегущие) координаты и растянутое время:

$$
\xi=x^{*}-c t^{*}, \quad \tau=\varepsilon t^{*},
$$

где $c$ - неизвестная безразмерная скорость волны. Тогда, разделив обе части 1-го уравнения (4) на

$$
\frac{1}{l} \frac{E h_{0}^{(i)}}{1-\mu_{0}^{2}}=\rho_{0} h_{0}^{(i)} c_{0}^{2} \frac{1}{l}
$$

получим:

$$
\begin{gathered}
\left\langle\frac{u_{m}}{l}\left[u_{1 \xi}^{(i)}-\mu_{0} \frac{w_{m} l}{u_{m} R^{(i)}} u_{3}^{(i)}+\frac{1}{2} \frac{u_{m}}{l} u_{1 \xi}^{(i)^{2}}+\frac{w_{m}^{2}}{u_{m} l} u_{3 \xi}^{(i)^{2}}+\frac{h_{0}^{(i)^{2}}}{12 l^{2}} \frac{w_{m}^{2}}{u_{m} l} u_{\xi \xi}^{(i)}{ }^{2}\right]\right\rangle_{\xi}- \\
-\frac{u_{m}}{l}\left(c^{2} u_{1 \xi \xi}^{(i)}-2 \varepsilon c u_{1 \xi \tau}^{(i)}+\varepsilon^{2} u_{1 \tau \tau}^{(i)}\right)=-\frac{l\left(q_{x}^{(i)}+\tilde{q}_{x}(i-1)\right)}{\rho_{0} h_{0}^{(i)} c_{0}^{2}} .
\end{gathered}
$$

Разделив обе части 2-го уравнения (4) на

$$
\frac{1}{R^{(i)}} \frac{E h_{0}^{(i)}}{1-\mu_{0}^{2}}=\frac{\rho_{0} h_{0}^{(i)} c_{0}^{2}}{R^{(i)}}
$$

получим:

$$
\begin{aligned}
& \frac{u_{m}}{l}\left\langle\frac{h_{0}^{(i)^{2}}}{12 l^{2}}\left[\frac{w_{m} R^{(i)}}{u_{m} l} u_{3 \xi \xi}^{(i)}\left(1+\frac{u_{m}}{l} u_{1 \xi}^{(i)}\right)\right]_{\xi \xi}+\right. \\
& +\left[-\mu_{0} u_{1 \xi}^{(i)}+\frac{w_{m} l}{u_{m} R^{(i)}} u_{3}^{(i)}-\mu_{0} \frac{1}{2} \frac{u_{m}}{l} u_{1 \xi}^{(i)^{2}}-\mu_{0} \frac{1}{2} \frac{w_{m}^{2}}{u_{m} l} u_{3 \xi}^{(i)^{2}}-\mu_{0} \frac{1}{2} \frac{h_{0}^{(i)}}{24 l^{2}} \frac{w_{m}^{2}}{u_{m} l} u_{3 \xi \xi}^{(i)}{ }^{2}\right]+ \\
& \left.+\frac{R^{(i)}}{l}\left\{\frac{w_{m}}{l} u_{3 \xi}^{(i)}\left[-u_{1 \xi}^{(i)}+\mu_{0} \frac{w_{m} l}{u_{m} R^{(i)}} u_{3}^{(i)}-\frac{1}{2} \frac{u_{m}}{l} u_{1 \xi}^{(i)^{2}}-\frac{w_{m}^{2}}{u_{m} l} u_{3 \xi}^{(i)^{2}}-\frac{1}{2} \frac{h_{0}^{(i)}{ }^{2}}{24 l^{2}} \frac{w_{m}^{2}}{u_{m} l} u_{3 \xi \xi}^{(i)}{ }^{2}\right]\right\}_{\xi}\right\rangle+ \\
& +\frac{w_{m} R^{(i)}}{l^{2}}\left(c^{2} u_{3 \xi \xi}^{(i)}-2 \varepsilon c u_{3 \xi \tau}^{(i)}+\varepsilon^{2} u_{3 \tau \tau}^{(i)}\right)=R^{(i)} \frac{(-1)^{i-1} q_{n}+\tilde{q}_{n}(i-1)}{\rho_{0} h_{0}^{(i)} c_{0}^{2}} .
\end{aligned}
$$

Разложим упругие перемещения по степеням $\varepsilon=u_{m} / l$ :

$$
u_{1}^{(i)}=u_{10}^{(i)}+\varepsilon u_{11}^{(i)}+\ldots, \quad u_{3}^{(i)}=u_{30}^{(i)}+\varepsilon u_{31}^{(i)}+\ldots
$$

подставим их в уравнения, разделим обе части уравнений на $\varepsilon=u_{m} / l$ и, оставляя члены $\varepsilon^{0}$ и $\varepsilon^{1}$, получим:

$$
\left(u_{10 \xi}^{(i)}-\mu_{0} \frac{w_{m} l}{u_{m} R^{(i)}} u_{30}^{(i)}\right)_{\xi}+\varepsilon\left\{u_{11 \xi \xi}^{(i)}-\mu_{0} \frac{w_{m} l}{u_{m} R^{(i)}} u_{31}^{(i)}+\frac{1}{2} \frac{u_{m}}{l \varepsilon} u_{10 \xi}^{(i)}\right\}_{\xi}-
$$




$$
\begin{gathered}
-c^{2} u_{10 \xi \xi}^{(i)}-\varepsilon c^{2} u_{11 \xi \xi}^{(i)}+2 \varepsilon c u_{10 \xi \tau}^{(i)}=-\frac{l^{2}\left(q_{x}^{(i)}+\tilde{q}_{x}(i-1)\right)}{u_{m} \rho_{0} h_{0}^{(i)} c_{0}^{2}} \\
-\mu_{0} u_{10 \xi}^{(i)}+\frac{w_{m} l}{u_{m} R^{(i)}} u_{30}^{(i)}+\varepsilon\left\langle-\mu_{0} u_{11 \xi}^{(i)}+\frac{w_{m} l}{u_{m} R^{(i)}} u_{31}^{(i)}-\frac{1}{2} \mu_{0} \frac{u_{m}}{l \varepsilon} u_{10 \xi}^{(i)}{ }^{2}\right\rangle+ \\
+\frac{w_{m} R^{(i)}}{u_{m} l} c^{2} u_{30 \xi \xi}^{(i)}=R^{(i)} l \frac{(-1)^{i-1} q_{n}+\tilde{q}_{n}(i-1)}{u_{m} \rho_{0} h_{0}^{(i)} c_{0}^{2}}
\end{gathered}
$$

Приравниваем к нулю коэффициенты при $\varepsilon^{0}$, получим систему уравнений

$$
\begin{gathered}
u_{10 \xi \xi}^{(i)}-\mu_{0} \frac{w_{m} l}{u_{m} R^{(i)}} u_{30 \xi}^{(i)}-c^{2} u_{10 \xi \xi}^{(i)}=0, \\
-\mu_{0} u_{10 \xi}^{(i)}+\frac{w_{m} l}{u_{m} R^{(i)}} u_{30}^{(i)}=0 .
\end{gathered}
$$

Из этой системы следует

$$
\frac{w_{m} l}{u_{m} R^{(i)}} u_{30}^{(i)}=\mu_{0} u_{10 \xi}^{(i)}, \quad\left(1-\mu_{0}^{2}-c^{2}\right) u_{10 \xi \xi}^{(i)}=0
$$

Следовательно, $u_{10}-$ произвольная функция, а безразмерная скорость волны $c=\sqrt{1-\mu_{0}^{2}}$, так как $c^{2}=1-\mu_{0}^{2}$. Приравниваем коэффициенты при $\varepsilon$ в правых и левых частях уравнений и учитываем предыдущие результаты, тогда получаем:

$$
\begin{gathered}
{\left[\mu_{0}^{2} u_{11 \xi}^{(i)}-\mu_{0} \frac{w_{m} l}{u_{m} R^{(i)}} u_{31}^{(i)}\right]_{\xi}+\frac{1}{2} \frac{u_{m}}{l \varepsilon} u_{10 \xi \xi}^{(i)}{ }^{2}+2 \sqrt{1-\mu_{0}^{2}} u_{10 \tau \xi}^{(i)}=-\frac{l^{2}}{\varepsilon u_{m} \rho_{0} h_{0}^{(i)} c_{0}^{2}}\left(q_{x}^{(i)}+\tilde{q}_{x}(i-1)\right),} \\
-\mu_{0} u_{11 \xi}+\frac{w_{m} l}{u_{m} R^{(i)}} u_{31}^{(i)}-\frac{1}{2} \mu_{0} \frac{u_{m}}{l \varepsilon}{u_{10 \xi}^{(i)}}^{2}+\frac{1}{\varepsilon} \frac{R^{(i)^{2}}}{l^{2}} \mu_{0}\left(1-\mu_{0}^{2}\right) u_{10 \xi \xi \xi}^{(i)}=R^{(i)} l \frac{(-1)^{i-1} q_{n}+\tilde{q}_{n}(i-1)}{\varepsilon u_{m} \rho_{0} h_{0}^{(i)} c_{0}^{2}} .
\end{gathered}
$$

Умножим обе части второго уравнения на $\mu_{0}$ и продифференцируем по $\xi$, получим систему

$$
\begin{aligned}
& \left(\mu_{0}^{2} u_{11 \xi}^{(i)}-\mu_{0} \frac{w_{m} l}{u_{m} R^{(i)}} u_{31 \xi}^{(i)}\right)_{\xi}+\frac{1}{2} \frac{u_{m}}{l \varepsilon} u_{10 \xi \xi}^{(i)}{ }^{2}=-\frac{l^{2}\left(q_{x}^{(i)}+\tilde{q}_{x}(i-1)\right)}{\varepsilon u_{m} \rho_{0} h_{0}^{(i)} c_{0}^{2}}, \\
& \left(\mu_{0}^{2} u_{11 \xi}^{(i)}-\mu_{0} \frac{w_{m} l}{u_{m} R^{(i)}} u_{31 \xi}^{(i)}\right)_{\xi}+\left[\frac{1}{2} \mu_{0}^{2} \frac{u_{m}}{l \varepsilon} u_{10 \xi}^{(i)}\right]_{\xi}+\frac{1}{\varepsilon} \frac{R^{(i)^{2}}}{l^{2}} \mu_{0}^{2}\left(1-\mu_{0}^{2}\right) u_{10 \xi \xi \xi \xi}^{(i)}= \\
& =\frac{R^{(i)} l}{\varepsilon u_{m} \rho_{0} h_{0}^{(i)} c_{0}^{2}} \mu_{0}\left((-1)^{i-1} \frac{\partial q_{n}}{\partial \xi}+(i-1) \frac{\partial \tilde{q}_{n}}{\partial \xi}\right) \text {. }
\end{aligned}
$$

Вычтем из первого уравнения второе и разделив обе части этого уравнения на $2 \sqrt{1-\mu_{0}^{2}}$, получим систему уравнений

$$
\begin{gathered}
u_{10 \xi \tau}^{(i)}+\frac{u_{m}}{l \varepsilon} \frac{\sqrt{1-\mu_{0}^{2}}}{2} u_{10 \xi}^{(i)} u_{10 \xi \xi}^{(i)}+\frac{1}{\varepsilon}\left(\frac{R^{(i)}}{l}\right)^{2} \frac{\mu_{0}^{2} \sqrt{1-\mu_{0}^{2}}}{2} u_{10 \xi \xi \xi \xi}^{(i)}= \\
=-\frac{1}{2 \sqrt{1-\mu_{0}^{2}}} \frac{l^{2}}{\varepsilon u_{m} \rho_{0} h_{0}^{(i)} c_{0}^{2}}\left[q_{x}^{(i)}+\tilde{q}_{x}(i-1)-\mu_{0} \frac{R^{(i)}}{l}\left(\frac{\partial q_{n}}{\partial \xi}(-1)^{i-1}+(i-1) \frac{\partial \tilde{q}_{n}}{\partial \xi}\right)\right] .
\end{gathered}
$$

В случае, когда жидкость отсутствует, правая часть уравнений становится равна нулю и получаются независимые уравнения КдВ. Надо определить правую часть, для чего необходимо решить уравнения гидродинамики для случая кольцевого и кругового сечений трубы.

\section{3. ОПРЕДЕЛЕНИЕ НАПРЯЖЕНИЙ, ДЕЙСТВУЮЩИХ НА ОБОЛОЧКИ СО СТОРОНЫ ВЯЗКОЙ НЕСЖИМАЕМОЙ ЖИДКОСТИ}

\section{1. Кольцевое сечение}

Введем безразмерные переменные и параметры:

$$
V_{r}=w_{m} \frac{c_{0}}{l} v_{r}, \quad V_{x}=w_{m} \frac{c_{0}}{\delta} v_{x}, \quad r=R_{2}+\delta r^{*}, \quad t^{*}=\frac{c_{0}}{l} t, \quad x^{*}=\frac{x}{l},
$$




$$
\begin{gathered}
p=\frac{\rho \nu c_{0} l w_{m}}{\delta^{3}} P+p_{0}, \quad \psi=\frac{\delta}{R_{2}}=o(1), \quad \lambda=\frac{w_{m}}{\delta}=\frac{w_{m}}{R_{2}} \frac{R_{2}}{\delta}=o\left(\frac{\varepsilon}{\psi}\right), \\
\frac{w_{m}}{R_{2}}=\frac{w_{m}}{\delta} \frac{\delta}{R_{2}}=\lambda \psi, \quad \frac{w_{m}}{l}=\frac{w_{m}}{\delta} \frac{\delta}{R_{i}} \frac{R_{i}}{l}=\lambda \psi \varepsilon^{1 / 2}, \quad \frac{\delta}{l}=\frac{\delta}{R_{i}} \frac{R_{i}}{l}=\psi \varepsilon^{1 / 2} .
\end{gathered}
$$

Во введенных безразмерных переменных получим уравнения гидродинамики:

$$
\begin{gathered}
\left(\frac{\delta}{l}\right)^{2}\left\{\frac{\delta c_{0}}{\nu} \frac{\delta}{l}\left[\frac{\partial v_{r}}{\partial t^{*}}+\lambda\left(v_{r} \frac{\partial v_{r}}{\partial r^{*}}+v_{x} \frac{\partial v_{r}}{\partial x^{*}}\right)\right]\right\}+\frac{\partial P}{\partial r^{*}}= \\
=\left(\frac{\delta}{l}\right)^{2}\left[\frac{\partial^{2} v_{r}}{\partial r^{* 2}}+\frac{\delta}{R_{2}\left(1+\psi_{r^{*}}\right)} \frac{\partial v_{r}}{\partial r^{*}}-\frac{\delta^{2} v_{r}}{R_{2}^{2}\left(1+\psi_{r^{*}}\right)^{2}}+\frac{\delta^{2}}{l^{2}} \frac{\partial^{2} v_{r}}{\partial x^{* 2}}\right], \\
\frac{\delta c_{0}}{\nu} \frac{\delta}{l}\left[\frac{\partial v_{x}}{\partial t^{*}}+\lambda\left(v_{r} \frac{\partial v_{r}}{\partial r^{*}}+v_{x} \frac{\partial v_{x}}{\partial x^{*}}\right)\right]+\frac{\partial P}{\partial x^{*}}=\frac{\partial^{2} v_{x}}{\partial r^{* 2}}+\frac{\delta}{R_{2}\left(1+\psi_{r^{*}}\right)} \frac{\partial v_{x}}{\partial r^{*}}+\frac{\delta^{2}}{l^{2}} \frac{\partial^{2} v_{x}}{\partial x^{* 2}}, ; \\
\frac{\partial v_{r}}{\partial r^{*}}+\frac{\delta v_{r}}{R_{2}\left(1+\psi_{r^{*}}\right)}+\frac{\partial v_{x}}{\partial x^{*}}=0 ;
\end{gathered}
$$

и граничные условия

$$
\begin{gathered}
\frac{\delta}{l} \frac{u_{m}}{w_{m}} \frac{\partial u_{1}^{(i)}}{\partial t^{*}}=v_{x}-\frac{u_{m}}{w_{m}} \frac{\delta}{l} \lambda u_{1}^{(i)} \frac{\partial v_{x}}{\partial x^{*}}-\lambda u_{3}^{(i)} \frac{\partial v_{x}}{\partial r^{*}}, \\
-\frac{\partial u_{3}^{(i)}}{\partial t^{*}}=v_{r}+\frac{u_{m}}{w_{m}} \frac{\delta}{l} \lambda u_{1}^{(i)} \frac{\partial v_{r}}{\partial x^{*}}-\lambda u_{3}^{(i)} \frac{\partial v_{r}}{\partial r^{*}}
\end{gathered}
$$

при $r^{*}=1-\lambda u_{3}^{(1)}$ и $r^{*}=-\lambda u_{3}^{(2)}$.

Полагая теперь $\delta / l=0, \delta / R_{2}=0$ (нулевое приближение по $\delta / l-$ гидродинамическая теория смазки), а также $\frac{\delta}{l} \frac{\delta c_{0}}{\nu}=0-$ ползущие течения, получим уравнения гидродинамики:

$$
\frac{\partial P}{\partial r^{*}}=0, \frac{\partial P}{\partial x^{*}}=\frac{\partial^{2} v_{x}}{\partial r^{* 2}}, \frac{\partial v_{r}}{\partial r^{*}}+\frac{\partial v_{x}}{\partial x^{*}}=0
$$

и граничные условия: $v_{r}=-\frac{\partial u_{3}^{(i)}}{\partial t^{*}}, v_{x}=0$ при $r^{*}=1-\lambda u_{3}^{(1)}$ и $r^{*}=-\lambda u_{3}^{(2)}$. Раскладывая давление и компоненты скорости по степеням малого параметра $\lambda$

$$
P=P^{0}+\lambda P^{1}+\ldots, \quad v_{r}=v_{r}^{0}+\lambda v_{r}^{1}+\ldots, \quad v_{x}=v_{x}^{0}+\lambda v_{x}^{1}+\ldots
$$

Для первых членов разложения получим те же уравнения

$$
\frac{\partial P^{0}}{\partial r^{*}}=0, \quad \frac{\partial P^{0}}{\partial x^{*}}=\frac{\partial^{2} v_{x}^{0}}{\partial r^{* 2}}, \quad \frac{\partial v_{r}^{0}}{\partial r^{*}}+\frac{\partial v_{x}^{0}}{\partial x^{*}}=0
$$

и граничные условия

$$
\begin{array}{llll}
v_{r}^{0}=-\frac{\partial u_{3}^{(1)}}{\partial t^{*}}, & v_{x}^{0}=0 & \text { при } & r^{*}=1, \\
v_{r}^{0}=-\frac{\partial u_{3}^{(2)}}{\partial t^{*}}, & v_{x}^{0}=0 & \text { при } & r^{*}=0 .
\end{array}
$$

С точностью до $\psi, \lambda$ получим:

$$
\begin{aligned}
& \cos \left(\widehat{\overline{-\bar{n}, \bar{n}_{r}}}\right) \approx 1, \quad \cos (\widehat{\overline{-\bar{n}, \bar{i}}}) \approx 0, \\
& q_{x}^{i}=\left.\left.\rho \nu \frac{w_{m} c_{0}}{\delta^{2}}\left[\frac{\partial v_{x^{*}}}{\partial r^{*}}+\frac{\delta^{2}}{l^{2}} \frac{\partial v_{r}^{*}}{\partial x^{*}}\right]\right|_{\substack{r_{2}^{*}=-\frac{w_{m} u_{3}^{(2)}}{\delta} \\
r_{1}^{*}=1-\frac{w_{m}}{\delta} u_{3}^{(1)}}} \approx \rho \nu \frac{w_{m} c_{0}}{\delta^{2}} \frac{\partial v_{x^{*}}}{\partial r^{*}}\right|_{\substack{r_{2}^{*}=-\lambda u_{3}^{(2)} \\
r_{1}^{*}=1-\lambda u_{3}^{(1)}}}, \\
& q_{n}=-\frac{\rho \nu c_{0} l w_{m}}{\delta^{3}} P-p_{0}+2 \rho \nu \frac{w_{m} c_{0}}{l \delta} \frac{\partial v_{r}}{\partial r^{*}}=
\end{aligned}
$$




$$
=-p_{0}-\frac{\rho \nu c_{0} l w_{m}}{\delta^{3}}\left(P-2 \frac{\delta^{2}}{l^{2}} \frac{\partial v_{r}}{\partial r^{*}}\right)=\left\{\left(\frac{\delta}{l}\right)^{2}=\psi_{i}^{2} \varepsilon_{i}\right\} \approx-p_{0}-\frac{\rho \nu c_{0} l w_{m}}{\delta^{3}} P .
$$

Из уравнений движения жидкости получаем с учетом граничных условий

$$
v_{x}^{0}=\frac{r^{* 2}-r^{*}}{2} \frac{\partial P^{0}}{\partial x^{*}}
$$

Подставляя в уравнение неразрывности, получим:

$$
\frac{\partial v_{r}^{0}}{\partial r^{*}}=-\frac{r^{* 2}-r^{*}}{2} \frac{\partial^{2} P^{0}}{\partial x^{* 2}}
$$

Тогда, учитывая условия при $r^{*}=0$, получаем:

$$
v_{r}^{0}=-\frac{\partial u_{3}^{(2)}}{\partial t^{*}}-\frac{1}{2}\left(\frac{r^{* 3}}{3}-\frac{r^{* 2}}{2}\right) \frac{\partial^{2} P^{0}}{\partial x^{* 2}} .
$$

Удовлетворяя условиям при $r^{*}=1$, найдем

$$
\begin{gathered}
\frac{\partial^{2} P^{0}}{\partial x^{* 2}}=12\left(\frac{\partial u_{3}^{(2)}}{\partial t^{*}}-\frac{\partial u_{3}^{(1)}}{\partial t^{*}}\right), \\
\frac{\partial P^{0}}{\partial x^{*}}=12 \int\left(\frac{\partial u_{3}^{(2)}}{\partial t^{*}}-\frac{\partial u_{3}^{(1)}}{\partial t^{*}}\right) d x^{*}, \\
P^{0}=12 \int\left[\int\left(\frac{\partial u_{3}^{(2)}}{\partial t^{*}}-\frac{\partial u_{3}^{(1)}}{\partial t^{*}}\right) d x^{*}\right] d x^{*} .
\end{gathered}
$$

При этом имеем:

$$
\begin{gathered}
v_{x}^{0}=\left(r^{* 2}-r^{*}\right) 6 \int\left(\frac{\partial u_{3}^{(2)}}{\partial t^{*}}-\frac{\partial u_{3}^{(1)}}{\partial t^{*}}\right) d x^{*} \\
\frac{\partial v_{x}^{0}}{\partial r^{*}}=\left(2 r^{*}-1\right) 6 \int\left(\frac{\partial u_{3}^{(2)}}{\partial t^{*}}-\frac{\partial u_{3}^{(1)}}{\partial t^{*}}\right) d x^{*} \\
\left.\frac{\partial v_{x}^{0}}{\partial r^{*}}\right|_{r^{*}=1}=6 \int\left(\frac{\partial u_{3}^{(2)}}{\partial t^{*}}-\frac{\partial u_{3}^{(1)}}{\partial t^{*}}\right) d x^{*} \\
\left.\frac{\partial v_{x}^{0}}{\partial r^{*}}\right|_{r^{*}=0}=6 \int\left(\frac{\partial u_{3}^{(1)}}{\partial t^{*}}-\frac{\partial u_{3}^{(2)}}{\partial t^{*}}\right) d x^{*}
\end{gathered}
$$

Учитывая, что введены переменные $\xi=x^{*}-c t^{*}$ и $\tau=\varepsilon t^{*}, c=\sqrt{1-\mu_{0}^{2}}$, найдем

$$
\begin{gathered}
P^{0}=12 \int\left[\int\left(c \frac{\partial u_{30}^{(1)}}{\partial \xi}-c \frac{\partial u_{30}^{(2)}}{\partial \xi}+\varepsilon c \frac{\partial u_{31}^{(1)}}{\partial \xi}-\varepsilon \frac{\partial u_{30}^{(1)}}{\partial \tau}-\varepsilon c \frac{\partial u_{31}^{(2)}}{\partial \xi}+\varepsilon \frac{\partial u_{30}^{(1)}}{\partial \tau}\right) d \xi\right] d \xi= \\
=12 \sqrt{1-\mu_{0}^{2}} \int\left(u_{30}^{(1)}-u_{30}^{(2)}\right) d \xi \\
-\frac{1}{\rho_{0} h_{0}^{(i)} c_{0}^{2}} q_{n}=\frac{\rho_{0} \nu c_{0} l w_{m}}{\delta^{3} \rho_{0} h_{0}^{(i)} c_{0}^{2}} 12 \sqrt{1-\mu_{0}^{2}} \int\left(u_{30}^{(1)}-u_{30}^{(2)}\right) d \xi \\
-\frac{1}{\rho_{0} h_{0}^{(i)} c_{0}^{2}} \frac{\partial q_{n}}{\partial \xi}=\frac{\rho \nu c_{0} l w_{m}}{\delta^{3} \rho_{0} h_{0}^{(i)} c_{0}^{2}} 12 \sqrt{1-\mu_{0}^{2}}\left(u_{30}^{(1)}-u_{30}^{(2)}\right)
\end{gathered}
$$

и учитывая, что $w_{m} l u_{30}^{(i)}=\mu_{0} u_{m} R^{(i)} u_{10 \xi}^{(i)}$, найдем

$$
\frac{1}{\rho_{0} h_{0}^{(i)} c_{0}^{2}} \frac{\partial q_{n}}{\partial \xi}=\frac{\rho \nu}{\delta^{3} \rho_{0} h_{0}^{(i)} c_{0} \mu_{0}} 12 \sqrt{1-\mu_{0}^{2}}\left[u_{m} R^{(2)} u_{10 \xi}^{(2)}-u_{m} R^{(1)} u_{10 \xi}^{(1)}\right], \quad q_{x}^{(i)}=\frac{\delta}{2 l} \frac{\partial q_{n}}{\partial \xi}(-1)^{i}
$$




\section{2. Круговое сечение}

Рассматривая круговое сечение, введем безразмерные переменные и параметры

$$
\begin{gathered}
V_{r}=w_{m} \frac{c_{0}}{l} v_{r}, \quad V_{x}=w_{m} \frac{c_{0}}{R_{3}} v_{x}, \quad r^{*}=\frac{r}{R_{3}}, \quad t^{*}=\frac{c_{0}}{l} t, \quad x^{*}=\frac{1}{l} x, \\
p=\frac{\tilde{\rho} \tilde{\nu} c_{0} l w_{m}}{R_{3}^{3}} P+p_{0}, \quad \frac{R_{3}}{l}=\psi=O\left(\varepsilon^{1 / 2}\right), \quad \lambda=\frac{w_{m}}{R_{3}}=O(\varepsilon) .
\end{gathered}
$$

В этих переменных получим уравнения гидродинамики:

$$
\begin{gathered}
\psi^{2}\left\{\psi \frac{R_{3} c_{0}}{\tilde{\nu}}\left[\frac{\partial v_{r}}{\partial t^{*}}+\lambda\left(v_{r} \frac{\partial v_{r}}{\partial r^{*}}+v_{x} \frac{\partial v_{r}}{\partial x^{*}}\right)\right]+\frac{\partial P}{\partial r^{*}}\right\}=\psi^{2}\left[\frac{\partial^{2} v_{r}}{\partial r^{* 2}}+\frac{1}{r^{*}} v_{r} \frac{\partial v_{r}}{\partial r^{*}}-\frac{\partial^{2} v_{r}}{\partial r^{* 2}}+\psi^{2} \frac{\partial^{2} v_{r}}{\partial x^{* 2}}\right], \\
\psi \frac{R_{3} c_{0}}{\tilde{\nu}}\left[\frac{\partial v_{x}}{\partial t^{*}}+\lambda\left(v_{r} \frac{\partial v_{x}}{\partial r^{*}}+v_{x} \frac{\partial v_{x}}{\partial x^{*}}\right)\right]+\frac{\partial P}{\partial x^{*}}=\frac{\partial^{2} v_{x}}{\partial r^{* 2}}+\frac{1}{r^{*}} \frac{\partial v_{x}}{\partial r^{*}}+\psi^{2} \frac{\partial^{2} v_{x}}{\partial x^{* 2}}, \\
\frac{\partial v_{r}}{\partial r^{*}}+\frac{v_{r}}{r^{*}}+\frac{\partial v_{x}}{\partial x^{*}}=0
\end{gathered}
$$

и граничные условия

$$
\begin{gathered}
\frac{u_{m} R_{3}}{w_{m} l} \frac{\partial u_{1}^{(2)}}{\partial t}=v_{x}+\frac{u_{m} R_{3}}{w_{m} l} \lambda u_{1}^{(2)} \frac{\partial v_{x}}{\partial x^{*}}-\lambda u_{3}^{(2)} \frac{\partial v_{x}}{\partial r^{*}} \\
-\frac{\partial u_{3}^{(2)}}{\partial t}=v_{r}+\frac{u_{m} R_{3}}{w_{m} l} \lambda u_{1}^{(2)} \frac{\partial v_{r}}{\partial x^{*}}-\lambda u_{3}^{(2)} \frac{\partial v_{r}}{\partial r^{*}}
\end{gathered}
$$

при $r^{*}=1-\lambda u_{3}^{(2)} ; v_{r}, v_{x}-$ ограничены при $r^{*}=0: r^{*} \frac{\partial v_{r}}{\partial r^{*}}=0, r^{*} \frac{\partial v_{x}}{\partial r^{*}}=0$. Полагая теперь $\psi=0$ (нулевое приближение по $\psi-$ гидродинамическая теория смазки [12]), а также $\psi \frac{R_{3} c_{0}}{\tilde{\nu}}=0-$ ползущие течения $[13,14]$, получаем уравнения гидродинамики

$$
\frac{\partial P}{\partial r^{*}}=0, \quad \frac{\partial P}{\partial x^{*}}=\frac{1}{r^{*}} \frac{\partial}{\partial r^{*}}\left(r^{*} \frac{\partial v_{x}}{\partial r^{*}}\right), \quad \frac{1}{r^{*}} \frac{\partial}{\partial r^{*}}\left(r^{*} v_{r}\right)+\frac{\partial v_{x}}{\partial x^{*}}=0 .
$$

Раскладывая давление и компоненты скорости по степеням малого параметра $\lambda$

$$
P=P^{0}+\lambda P^{1}+\ldots, \quad v_{x}=v_{x}^{0}+\lambda v_{x}^{1}+\ldots, \quad v_{r}=v_{r}^{0}+\lambda v_{r}^{1}+\ldots
$$

для первых членов разложений получим те же уравнения

$$
\frac{\partial P^{0}}{\partial r^{*}}=0, \quad \frac{\partial P^{0}}{\partial x^{*}}=\frac{1}{r^{*}} \frac{\partial}{\partial r^{*}}\left(r^{*} \frac{\partial v_{x}^{0}}{\partial r^{*}}\right), \quad \frac{1}{r^{*}} \frac{\partial}{\partial r^{*}}\left(r^{*} v_{r}^{0}\right)+\frac{\partial v_{x}^{0}}{\partial x^{*}}=0
$$

и граничные условия вида

$$
\begin{array}{cc}
v_{r}^{0}=-\frac{\partial u_{3}^{(2)}}{\partial t^{*}}, & v_{x}^{0}=\frac{u_{m} R_{3}}{w_{m} l} \frac{\partial u_{1}^{(2)}}{\partial t^{*}} \quad \text { при } \quad r^{*}=1, \\
r^{*} \frac{\partial v_{r}^{0}}{\partial r^{*}}=0, & r^{*} \frac{\partial v_{x}^{0}}{\partial r^{*}}=0 \quad \text { при } \quad r^{*}=0 .
\end{array}
$$

Определим теперь в этих переменных напряжения со стороны жидкости на оболочке. С точностью до $\lambda, \psi$ имеем

$$
\begin{gathered}
\cos \left(\widehat{\bar{n}, \bar{n}_{r}}\right)=\frac{R_{3}\left(1-\lambda u_{3}^{(2)}\right)}{R_{3}\left(1-\lambda u_{3}^{(2)}\right)\left(1+\lambda \psi \frac{\partial u_{3}^{(2)}}{\partial x^{*}}\right)} \approx 1, \\
\cos (\widehat{\bar{n}, \bar{i}})=-\frac{\lambda \psi \frac{\partial u_{3}^{(2)}}{\partial x^{*}} R_{3}\left(1-\lambda u_{3}^{(2)}\right)}{R_{3}\left(1-\lambda u_{3}^{(2)}\right)\left(1+\lambda \psi \frac{\partial u_{3}^{(2)}}{\partial x^{*}}\right)} \approx 0,
\end{gathered}
$$




$$
\begin{gathered}
\tilde{q}_{x}=\left.\left.\tilde{\rho} \tilde{\nu} \frac{c_{0} w_{m}}{R_{3}^{2}}\left[\frac{\partial v_{x}}{\partial r^{*}}+\psi^{2} \frac{\partial v_{r}}{\partial x^{*}}\right]\right|_{r^{*}=1-\lambda u_{3}^{(2)}} \approx \frac{w_{m}}{R_{3}} \frac{\tilde{\nu}}{R_{3} c_{0}} \tilde{\rho} c_{0}^{2} \frac{\partial v_{x}}{\partial r^{*}}\right|_{r^{*}=1}, \\
\tilde{q}_{n}=\left.\left[-p_{0}-\frac{\tilde{\rho} \tilde{\nu} c_{0} w_{m} l}{R_{3}^{3}} P+2 \tilde{\rho} \tilde{\nu} \frac{w_{m} c_{0}}{l R_{3}} \frac{\partial v_{r}}{\partial r^{*}}\right]\right|_{r^{*}=1-\lambda u_{3}^{(2)}} \approx-p_{0}-\frac{w_{m}}{R_{3}}\left(\frac{l}{R}\right) \frac{\tilde{\nu}}{R_{3} c_{0}} \tilde{\rho} c_{0}^{2} P .
\end{gathered}
$$

Получаем:

$$
\tilde{q}_{x}=\left.\lambda \frac{\tilde{\nu}}{R_{3} c_{0}} \tilde{\rho} c_{0}^{2} \frac{\partial v_{x}}{\partial r^{*}}\right|_{r^{*}=1}, \quad \tilde{q}_{n}=-p_{0}-\frac{\lambda}{\psi} \frac{\tilde{\nu}}{R_{3} c_{0}} \tilde{\rho}_{0} c_{0}^{2} P .
$$

Решение уравнений гидродинамики легко получить (это классические уравнения гидродинамической теории смазки). Из уравнений движения имеем:

$$
v_{x}=\frac{u_{m} R_{3}}{w_{m} l} \frac{\partial u_{1}^{(2)}}{\partial t^{*}}+\frac{r^{* 2}-1}{4} \frac{\partial P}{\partial x^{*}}, \quad \frac{\partial v_{x}}{\partial x^{*}}=\frac{u_{m} R_{3}}{w_{m} l} \frac{\partial^{2} u_{1}^{(2)}}{\partial x^{*} \partial t^{*}}+\frac{r^{* 2}-1}{4} \frac{\partial^{2} P}{\partial x^{* 2}} .
$$

Здесь учтены граничные условия при $r^{*}=1, r^{*}=0$. Подставляя в уравнение неразрывности, получаем:

$$
\frac{1}{r^{*}} \frac{\partial}{\partial r^{*}}\left(r^{*} v_{r}^{*}\right)=-\frac{u_{m} R_{3}}{w_{m} l} \frac{\partial^{2} u_{1}^{(2)}}{\partial x^{*} \partial t^{*}}-\frac{r^{* 2}-1}{4} \frac{\partial^{2} P}{\partial x^{* 2}} .
$$

Тогда, учитывая условия при $r^{*}=0$, получим:

$$
v_{r}=-\frac{r^{*}}{2} \frac{u_{m} R_{3}}{w_{m} l} \frac{\partial^{2} u_{1}^{(2)}}{\partial x^{*} \partial t^{*}}-\frac{1}{4}\left(\frac{r^{* 3}}{4}-\frac{r^{*}}{2}\right) \frac{\partial^{2} P}{\partial x^{* 2}} .
$$

Удовлетворяя граничным условиям при $r^{*}=1$, найдем

$$
\frac{\partial^{2} P}{\partial x^{* 2}}=16\left[\frac{1}{2} \frac{u_{m} R_{3}}{w_{m} l} \frac{\partial^{2} u_{1}^{(2)}}{\partial x^{*} \partial t^{*}}-\frac{\partial u_{3}^{(2)}}{\partial t^{*}}\right] .
$$

Интегрируя, получим:

$$
\frac{\partial P}{\partial x^{*}}=16 \int\left[\frac{1}{2} \frac{u_{m} R_{3}}{w_{m} l} \frac{\partial^{2} u_{1}^{(2)}}{\partial x^{*} \partial t^{*}}-\frac{\partial u_{3}^{(2)}}{\partial t^{*}}\right] d x^{*}=16 \frac{\partial}{\partial t^{*}}\left[\frac{1}{2} \frac{u_{m} R_{3}}{w_{m} l} u_{1}^{(2)}-\int u_{3}^{(2)} d x^{*}\right]
$$

и

$$
\begin{gathered}
P=16 \int \frac{\partial}{\partial t^{*}}\left[\frac{1}{2} \frac{u_{m} R_{3}}{w_{m} l} u_{1}^{(2)}-\int u_{3}^{(2)} d x^{*}\right] d x^{*}, \\
\left.\frac{\partial v_{x}}{\partial r^{*}}\right|_{r^{*}=1}=\left.\frac{r^{*}}{2} \frac{\partial}{\partial t^{*}} 16\left[\frac{1}{2} \frac{u_{m} R_{3}}{w_{m} l} u_{1}^{(2)}-\int u_{3}^{(2)} d x^{*}\right]\right|_{r^{*}=1}=8 \frac{\partial}{\partial t^{*}}\left[\frac{1}{2} \frac{u_{m} R_{3}}{w_{m} l} u_{1}^{(2)}-\int u_{3}^{(2)} d x^{*}\right] .
\end{gathered}
$$

Учитывая, что введены переменные $\xi=x^{*}-c t^{*}$ и $\tau=\varepsilon t^{*}, c=\sqrt{1-\mu_{0}^{2}}$, найдем с точностью до $\varepsilon$ и с учетом связи (8)

$$
P=8 \sqrt{1-\mu_{0}^{2}} \frac{u_{m} R_{3}}{w_{m} l}\left\{2 \mu_{0} \frac{R^{(2)}}{R_{3}}-1\right\} u_{10}^{(2)} .
$$

При этом

$$
\left.\frac{\partial v_{x}}{\partial r^{*}}\right|_{r^{*}=1}=4 \sqrt{1-\mu_{0}^{2}} \frac{u_{m} R_{3}}{w_{m} l}\left\{2 \mu_{0} \frac{R^{(2)}}{R_{3}}-1\right\} \frac{\partial u_{10}^{(2)}}{\partial \xi} .
$$

Тогда, учитывая, что $\frac{w_{m} l}{u_{m} R_{3}} u_{30}^{(2)}=\mu_{0} u_{10 \xi}$ получаем:

$$
\tilde{q}_{x}-\mu_{0} \frac{R^{(2)}}{l} \frac{\partial \tilde{q}_{n}}{\partial \xi}=-\frac{\tilde{\nu}}{R_{3} c_{0}} \tilde{\rho} c_{0}^{2} 4 \sqrt{1-\mu_{0}^{2}} \frac{u_{m}}{l}\left[1-\left(2 \mu_{0} \frac{R^{(2)}}{R_{3}}\right)^{2}\right] \frac{\partial u_{10}^{(2)}}{\partial \xi} .
$$




\section{4. УРАВНЕНИЕ ДИНАМИКИ ОБОЛОЧКИ}

Система уравнений становится такой с учетом найденной правой частью

$$
\begin{gathered}
u_{10 \xi \tau}^{(1)}+\frac{u_{m}}{l \varepsilon} \frac{\sqrt{1-\mu_{0}^{2}}}{2} u_{10 \xi}^{(1)} u_{10 \xi \xi}^{(1)}+\frac{1}{\varepsilon}\left(\frac{R}{l}\right)^{2} \frac{\mu_{0}^{2} \sqrt{1-\mu_{0}^{2}}}{2} u_{10 \xi \xi \xi \xi}^{(1)}+ \\
+6 \mu_{0}^{2} \frac{\rho l}{\rho_{0} h_{0}} \frac{\nu}{R c_{0} \varepsilon}\left(\frac{R}{\delta}\right)^{3}\left[1+\frac{\delta}{2 \mu_{0} R}\right]\left[u_{10 \xi}^{(1)}-u_{10 \xi}^{(2)}\right]=0, \\
u_{10 \xi \tau}^{(2)}+\frac{u_{m}}{l \varepsilon} \frac{\sqrt{1-\mu_{0}^{2}}}{2} u_{10 \xi}^{(2)} u_{10 \xi \xi}^{(2)}+\frac{1}{\varepsilon}\left(\frac{R}{l}\right)^{2} \frac{\mu_{0}^{2} \sqrt{1-\mu_{0}^{2}}}{2} u_{10 \xi \xi \xi \xi}^{(2)}+ \\
+6 \mu_{0}^{2} \frac{\rho l}{\rho_{0} h_{0}} \frac{\nu}{R c_{0} \varepsilon}\left(\frac{R}{\delta}\right)^{3}\left[1+\frac{\delta}{2 \mu_{0} R}\right]\left[u_{10 \xi}^{(2)}-u_{10 \xi}^{(1)}\right]-2\left(1-4 \mu_{0}^{2}\right) \frac{\tilde{\rho} l}{\rho_{0} h_{0}} \frac{\tilde{\nu}}{R c_{0} \varepsilon} u_{10 \xi}^{(2)}=0 .
\end{gathered}
$$

Здесь с принятой точностью $h_{0} / R \approx O(\varepsilon), \delta / R_{2}=\psi \ll 1$ обозначено $R^{(1)} \approx R^{(2)}=R$, при этом положено $h_{0}^{(1)} \approx h_{0}^{(2)} \approx h_{0}$.

Можно также ввести обозначения $u_{10 \xi}^{(1)}=c_{3} \phi^{(1)}, u_{10 \xi}^{(2)}=c_{3} \phi^{(2)}, \eta=c_{1} \xi, t=c_{2} \tau$, где

$$
\begin{gathered}
c_{2}=6 \mu_{0}^{2} \frac{\rho l}{\rho_{0} h_{0} \varepsilon}\left(\frac{R}{\delta}\right)^{2}\left[1+\frac{\delta}{2 \mu_{0} R}\right] \frac{\nu}{\delta c_{0}}, \quad c_{1}=\left[c_{2} \varepsilon\left(\frac{l}{R}\right)^{2} \frac{2}{\mu_{0}^{2} \sqrt{1-\mu_{0}^{2}}}\right]^{1 / 3}, \\
c_{3}=\frac{c_{2}}{c_{1}} \frac{l_{\varepsilon}}{u_{m}} \frac{2}{\sqrt{1-\mu_{0}^{2}}} 6 \sigma_{0}, \quad \sigma=\frac{1-4 \mu_{0}^{2}}{3 \mu_{0}^{2}}\left(\frac{\delta}{R}\right)^{3} \frac{\tilde{\rho} \tilde{\nu}}{\rho \nu}\left[1+\frac{\delta}{2 \mu_{0} R}\right]^{-1},
\end{gathered}
$$

и получаем систему уравнений

$$
\begin{gathered}
\phi_{t}^{(1)}+6 \sigma_{0} \phi^{(1)} \phi_{\eta}^{(1)}+\phi_{\eta \eta \eta}^{(1)}+\phi^{(1)}-\phi^{(2)}=0, \\
\phi_{t}^{(2)}+6 \sigma_{0} \phi^{(2)} \phi_{\eta}^{(2)}+\phi_{\eta \eta \eta}^{(2)}+\phi^{(2)}-\phi^{(1)}-\sigma \phi^{(2)}=0 .
\end{gathered}
$$

Система уравнений (9) имеет в качестве точного решения при $\sigma=0$ (отсутствие жидкости во внутренней оболочке) следующее решение:

$$
\phi^{(1)}=\phi^{(2)}=\frac{2 k^{2}}{\cosh ^{2}\left(k x-4 k^{3} t\right)} .
$$

\section{5. ЧИСЛЕННОЕ МОДЕЛИРОВАНИЕ}

В работах [15-17] развит подход к построению разностных схем, основанный на построении переопределенной системы разностных уравнений, получаемой из аппроксимации интегральных законов сохранения и интегральных соотношений, связывающих искомые функции и их производные. В результате разностная схема определяется как условие совместности для данной системы. Таким образом, получается разностная схема, автоматически обеспечивающая выполнение интегральных законов сохранения по областям, составленным из шаблонов интегрирования построения.

Запишем систему уравнений (9) в интегральной форме:

$$
\begin{gathered}
\oint_{\partial \Omega}\left(-3 \sigma_{0} \phi^{(1)^{2}}-\phi_{\eta \eta}^{(1)}\right) d t+\phi^{(1)} d \eta+\iint_{\Omega}\left(\phi^{(1)}-\phi^{(2)}\right) d t d \eta=0, \\
\oint_{\partial \Omega}\left(-3 \sigma_{0} \phi^{(2)^{2}}-\phi_{\eta \eta}^{(2)}\right) d t+\phi^{(2)} d \eta+\iint_{\Omega}\left(\phi^{(2)}-\phi^{(1)}-\sigma \phi^{(2)}\right) d t d \eta=0
\end{gathered}
$$

для любой области $\Omega$.

Для перехода к дискретной формулировке сопоставим $u^{(i)^{n}}=\phi^{(i)}\left(t_{n}, \eta_{j}\right)$ и выберем в качестве 
базового контур, показанный на рис. 2. Добавим интегральные соотношения:

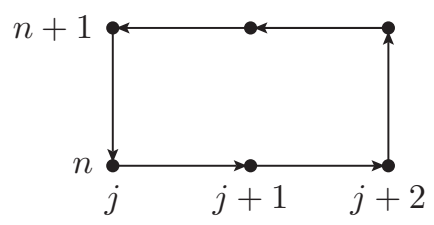

Рис. 2. Базовой контур для уравнения (11)

$$
\begin{gathered}
\int_{\eta_{j}}^{\eta_{j+1}} u_{\eta}^{(i)} d \eta=u^{(i)}\left(t, \eta_{j+1}\right)-u^{(i)}\left(t, \eta_{j}\right), \\
\int_{\eta_{j}}^{\eta_{j+2}} u^{(i)}{ }_{\eta \eta} d \eta=u^{(i)}\left(t, \eta_{j+2}\right)-u^{(i)}\left(t, \eta_{j}\right) .
\end{gathered}
$$

Используя для интегрирования по времени и первой производной по $\eta$ формулу трапеций, а по второй производной по $\eta$ формулу среднего значения и полагая $t_{n+1}-t_{n}=\tau, \eta_{j+1}-\eta_{j}=h$, перепишем соотношения (11), (12) в виде

$$
\begin{aligned}
& \left(-3 \sigma_{0}\left(u^{(1)^{2}}{ }_{j}^{n}+u_{j}^{(1)^{2 n+1}}-u_{j+2}^{(1)^{2}}{ }_{j+2}^{n}{ }_{j+2}^{(1)^{2 n+1}}\right)-\left(u_{\eta \eta_{j}}^{(1)^{n}}+u_{\eta \eta_{j}}^{(1)^{n+1}}-u_{\eta \eta_{j+2}^{(1)^{n}}}^{n}-u_{\eta \eta}^{(1)^{n+1}}\right)\right) \cdot \frac{\tau}{2}+ \\
& +\left(u^{(1)}{ }_{j+1}^{n+1}-u^{(1)^{n}}{ }_{j+1}\right) \cdot 2 h+\left(\left(u^{(1)^{n+1}}+u_{j+1}^{(1)^{n}}\right)-\left(u_{j+1}^{(2)^{n+1}}+u^{(2)^{n}}{ }_{j+1}\right)\right) \cdot h \tau=0, \\
& \left(-3 \sigma_{0}\left(u_{j}^{(2)^{2}}{ }_{j}^{n}+u_{j}^{(2)^{2 n+1}}-u_{j+2}^{(2)^{2 n}}-u_{j+2}^{(2)^{2 n+1}}\right)-\right. \\
& \left.-\left(u_{\eta \eta_{j}}^{(2)^{n}}+u_{\eta \eta_{j}}^{(2)^{n+1}}-u_{\eta \eta_{j+2}^{(2)}}^{n}-u_{\eta \eta_{j+2}^{(2)}}^{n+1}\right)\right) \cdot \frac{\tau}{2}+\left(u_{j+1}^{(2)^{n+1}}-u_{j+1}^{(2)^{n}}\right) \cdot 2 h+
\end{aligned}
$$

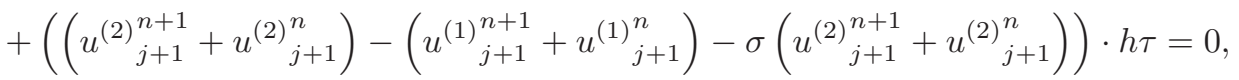

$$
\begin{aligned}
& \left(u_{\eta}^{(i)^{n}}{ }_{j+1}+u_{\eta}^{(i)}{ }_{j}^{n}\right) \cdot \frac{h}{2}=u_{j+1}^{(i)^{n}}-u_{j}^{(i)^{n}}, \\
& u_{\eta \eta_{j+1}}^{(i)^{n}} \cdot 2 h=u_{\eta}^{(i)^{n}{ }_{j+2}^{n}}-u_{\eta}^{(i)^{n}}{ }_{j}^{n} .
\end{aligned}
$$

Поскольку пакет [17] работает только в случае линейных разностных идеалов, а исходное дифференциальное уравнение (9) нелинейно, заменим нелинейную часть введением дополнительной функции $F^{(i)}=3 \sigma_{0} u^{(i)^{2}}$. За счет выбора допустимого упорядочения, так чтобы $u^{(1)} \succ$ $\succ u^{(2)} \succ \ldots \succ F^{(1)} \succ F^{(1)}$, а затем по переменным $n, j$, нелинейная часть не будет входить в лидирующие мономы системы при построении базиса Грёбнера, и структура базиса позволит проверить принадлежность искомой разностной схеме.

В результате получим следующую разностную схему для уравнения (9), аналогичную схеме Кранка - Николсона для уравнения теплопроводности:

$$
\begin{aligned}
& \frac{u_{j}^{(1)^{n+1}}-u_{j}^{(1)^{n}}}{\tau}+3 \sigma_{0} \frac{\left(u^{(1)^{2}}{ }_{j+1}^{n+1}-u^{(1)^{2}}{ }_{j-1}^{n+1}\right)+\left(u^{(1)^{2}}{ }_{j+1}^{n}-u^{(1)^{2 n}}{ }_{j-1}\right)}{4 h}+ \\
& +\frac{\left(u^{(1)}{ }_{j+2}^{n+1}-2 u^{(1)}{ }_{j+1}^{n+1}+2 u^{(1)}{ }_{j-1}^{n+1}-u^{(1)}{ }_{j-2}^{n+1}\right)+\left(u^{(1)}{ }_{j+2}^{n}-2 u^{(1)^{n}}{ }_{j+1}^{n}+2 u^{(1)_{j-1}^{n}}-u^{(1)}{ }_{j-2}^{n}\right)}{4 h^{3}}+ \\
& +\frac{u_{j}^{(1)}{ }_{j}^{n+1}+u_{j}^{(1)^{n}}}{2}-\frac{u_{j}^{(2)_{j}^{n+1}}+u_{j}^{(2)_{j}^{n}}}{2}=0, \\
& \frac{u_{j}^{(2)_{j}^{n+1}}-u_{j}^{(2)^{n}}}{\tau}+3 \sigma_{0} \frac{\left(u^{(2)^{2}}{ }_{j+1}^{n+1}-u^{(2)^{2}}{ }_{j-1}^{n+1}\right)+\left(u^{(2)^{2}}{ }_{j+1}^{n}-u^{(2)^{2}}{ }_{j-1}^{n}\right)}{4 h}+ \\
& +\frac{\left(u^{(2)}{ }_{j+2}^{n+1}-2 u^{(2)^{n+1}}+2 u^{(2)}{ }_{j-1}^{n+1}-u^{(2)_{j-2}^{n+1}}\right)+\left(u^{(2)_{j+2}^{n}}-2 u^{(2)_{j+1}^{n}}+2 u^{(2)_{j-1}^{n}}-u^{(2)}{ }_{j-2}^{n}\right)}{4 h^{3}}- \\
& -\frac{u^{(2)_{j}^{n+1}}+u^{(2)_{j}^{n}}}{2}-\frac{u^{(1)_{j}^{n+1}}+u^{(1)_{j}^{n}}}{2}-\sigma \frac{u_{j}^{(2)_{j}^{n+1}}+u_{j}^{(2)}{ }_{j}^{n}}{2}=0 .
\end{aligned}
$$

Полученные неявные разностные схемы имеют квадратичную и кубическую нелинейность для следующего временного слоя. При построении решения использована следующая линеаризация:

$$
v_{k+1}^{2}=v_{k+1}^{2}-v_{k}^{2}+v_{k}^{2}=\left(v_{k+1}-v_{k}\right)\left(v_{k+1}+v_{k}\right)+v_{k}^{2} \approx v_{k+1} \cdot 2 v_{k}-v_{k}^{2} .
$$


Количество итераций для достижения точности $10^{-12}$ на следующем временном слое, как правило, не превышало $2-3$. Шаг по времени $t$ брался равным половине шага по переменной $\eta$. Программа расчета была написана на языке Python с использованием пакета SciPy (http:\\scipy.org).

При отсутствии жидкости во внутренней оболочке, как показано в работе [7], возникает нелинейная волна деформации во внутренней оболочке, в которой ее не было в начальный момент времени, и амплитуды волн деформации в соосных оболочках со временем начинают совпадать. Эти амплитуды в два раза меньше исходной амплитуды волны деформации внешней оболочки в начальный момент времени.

Выполненные вычислительные эксперименты на рис. 3 позволили оценить влияние вязкой несжимаемой жидкости во внутренней оболочке на поведение нелинейной волны деформации при значении параметра $\sigma>0$.
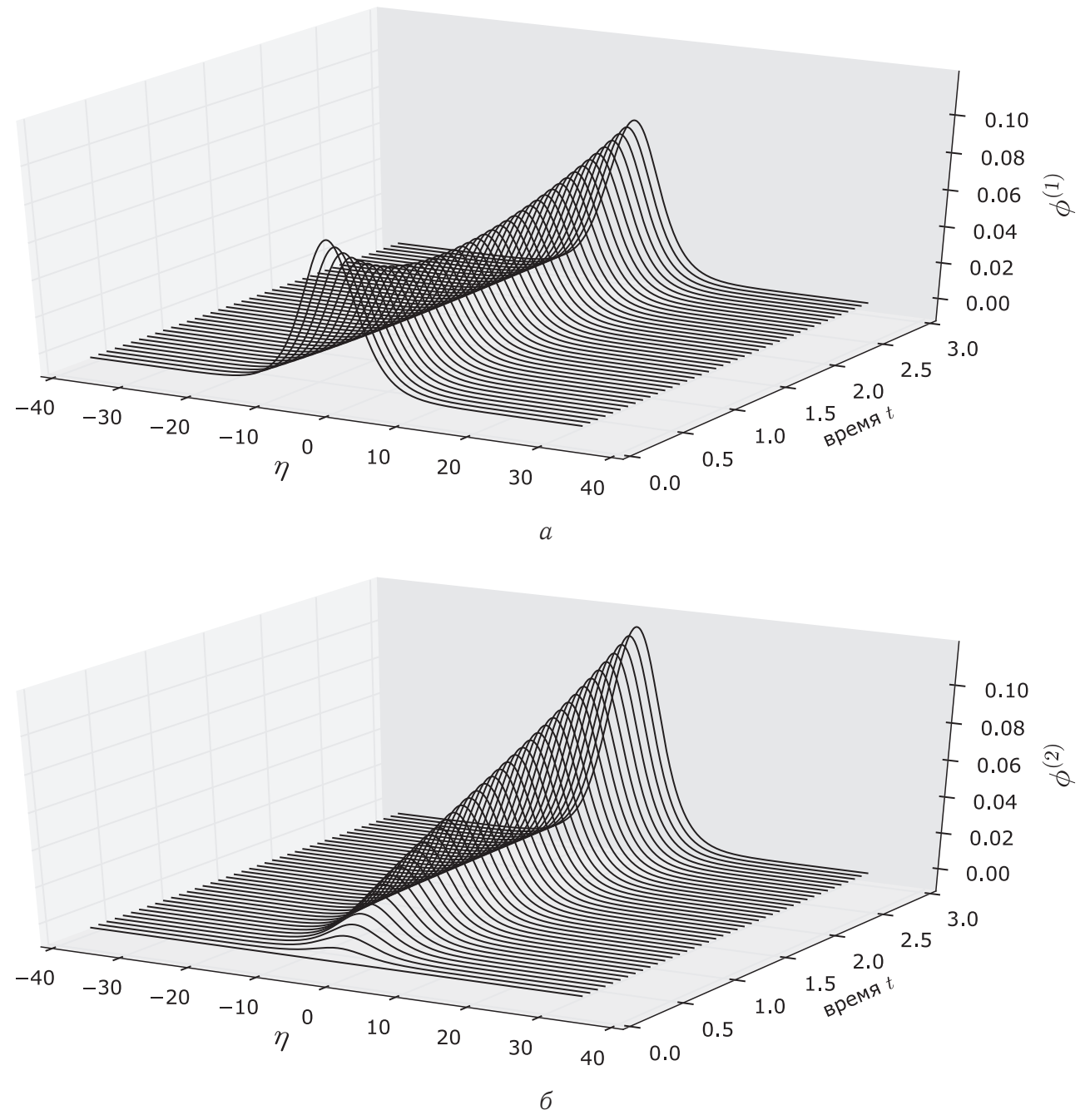

Рис. 3. Графики численного решения уравнений (9) при $\sigma=0.7$ с начальным условием $\phi^{(2)}=0$ и с $\phi^{(1)}$, взятого из точного решения (10) при $t=0 \mathrm{c} k=0.2: a-$ амплитуда волны для внешней оболочки; $\sigma$ - амплитуда волны для внутренней оболочки

Сначала происходит выравнивание амплитуд с их дальнейшим линейным ростом, при этом угол наклона амплитуды волны больше во внутренней оболочке. Наблюдается линейный синхронный рост амплитуды волны относительно времени в обеих оболочках при более сильном росте во внутренней оболочке.

Работа выполнена при финансовой поддержке РФФИ (проект № 16-01-00175-а). 


\section{Библиографический список}

1. Громека И. С. К теории движения жидкости в узких цилиндрических трубах // Громека И. С. Собрание сочинений. М. : Изд-во АН СССР, 1952. C. $149-171$.

2. Землянухин А. И., Могилевич Л. И. Нелинейные волны деформаций в цилиндрических оболочках // Изв. вузов. Прикладная нелинейная динамика. 1995. Т. 3, № 1. С. 52-58.

3. Ерофеев В. И., Клюева Н. В. Солитоны и нелинейные периодические волны деформации в стержнях, пластинах и оболочках (обзор) // Акустический журн. 2002. Т. 48, № 6. С. 725-740.

4. Землянухин А. И., Могилевич Л. И. Нелинейные волны в неоднородных цилиндрических оболочках : новое эволюционное уравнение // Акустический журн. 2001. Т. 47, № 3. С. 359-363.

5. Аршинов Г. А., Землянухин А. И., Могилевич Л. И. Двумерные уединенные волны в нелинейной вязкоупругой деформируемой среде // Акустический журн. 2000. Т. 46, № 1. С. 116-117.

6. Блинкова А. Ю., Иванов С. В., Ковалев А. Д., Могилевич Л. И. Математическое и компьютерное моделирование динамики нелинейных волн в физически нелинейных упругих цилиндрических оболочках, содержащих вязкую несжимаемую жидкость // Изв. Сарат. ун-та. Нов. сер. Сер. Физика. 2012. Т. 12, вып. 2. С. 12-18.

7. Блинков Ю. А., Ковалева И. А., Могилевич Л. И. Моделирование динамики нелинейных волн в соосных геометрически и физически нелинейных оболочках, содержащих вязкую несжимаемую жидкость между ними // Вестн. РУДН. Сер. Матем., информ., физ. 2013. Т. 3. С. 42-51.

8. Лойцяяский Л. Г. Механика жидкости и газа. М. : Дрофа, 2003. 840 с.

9. Валландер С. В. Лекции по гидроаэромеханике. Л. : Изд-во ЛГУ, 1978. 296 с.

10. Вольмир А. С. Нелинейная динамика пластинок и оболочек. М. : Наука, 1972. 432 с.

11. Вольмир A. С. Оболочки в потоке жидкости и газа : задачи гидроупругости. М. : Наука, 1979. $320 \mathrm{c}$.

12. Шлихтинг Г. Теория пограничного слоя. М. : Наука, 1974. 712 с.

13. Чивилихин С. А., Попов И. Ю., Гусаров В. В. Динамика скручивания нанотрубок в вязкой жидкости // Докл. АН. 2007. Т. 412, № 2. С. 201-203.

14. Попов И. Ю., Родыгина О. А., Чивилихин С. А., Гусаров В. В. Солитон в стенке нанотрубки и стоксово течение в ней // Письма в ЖТФ. 2010. Т. 36, № 18. С. $48-54$.

15. Гердт В. П., Блинков Ю. А. О стратегии выбора немультипликативных продолжений при вычислении базисов Жане // Программирование. 2007. T. 33, № 3. С. 34-43.

16. Блинков Ю. А., Гердт В. П. Специализированная система компьютерной алгебры GINV // Программирование. 2008. Т. 34, № 2. С. 67-80.

17. Gerdt V. P., Blinkov Yu. A. Involution and difference schemes for the Navier - Stokes equations // Computer Algebra in Scientific Computing. Vol. 5743 of Lecture Notes in Computer Science. 2009. P. 94105. DOI: 10.1007/978-3-642-04103-7_10.

\title{
Wave Occurrences Mathematical Modeling in Two Geometrically Nonlinear Elastic Coaxial Cylindrical Shells, Containing Viscous Incompressible Liquid
}

\section{Yu. A. Blinkov ${ }^{1}$, A. V. Mesyanzhin ${ }^{2}$, L. I. Mogilevich ${ }^{3}$}

\author{
${ }^{1}$ Yury A. Blinkov, Saratov State University, 83, Astrakhanskaya st., 410012, Saratov, Russia, BlinkovUA@info.sgu.ru \\ ${ }^{2}$ Artem V. Mesyanzhin, Industrial Automatics Design Bureau JSC, 239, B. Sadovaya st., 410005, Saratov, Russia, \\ a.v.mesyanzhin@gmail.com \\ ${ }^{3}$ Lev I. Mogilevich, University of Means of Communication Emperor Nicholas II (Volga Region Branch of Moscow State), 1a, \\ Astrakhanskaya st., 410012, Saratov, Russia, mogilevich@sgu.ru
}

The investigation of deformation waves behavior in elastic shells is one of the important trends in the contemporary wave dynamics. There exist mathematical models of wave motions in infinitely long geometrically non-linear shells, containing viscous incompressible liquid, based on the related hydroelasticity problems, which are derived by the shell dynamics and viscous incompressible liquid equations in the form of generalized Korteweg - de Vries equations. In addition, mathematical models of the wave process in infinitely long geometrically non-linear coaxial cylindrical elastic shells are obtained by the perturbation method. These models differ from the known ones by the consideration of incompressible liquid between the shells, based on the related hydroelasticity problems. These problems are described by shell dynamics and viscous incompressible liquid equations with corresponding edge conditions in the form of generalized KdV equation system. The paper presents the investigation of wave occurrences in two geometrically non-linear elastic coaxial cylindrical shells of Kirchhoff-Love type, containing viscous incompressible liquid both between and inside them. The difference schemes of Crank-Nicholson type are obtained for the considered equation system by taking into account liquid and with 
the help of Gröbner basis construction. To generate these difference schemes, the basic integral difference correlations, approximating the initial equation system, were used. The usage of Gröbner basis technology provides generating the schemes, for which it becomes possible to obtain discrete analogs of the laws of preserving the initial equation system. To do that, equivalent transformations were made. Based on the computation algorithm the corresponding software, providing graphs generation and numerical solutions under exact solutions of coaxial shell dynamics equation system obtaining, was developed.

Key words: nonlinear waves, viscous incompressible liquid, elastic cylindrical shells.

This work was supported by the Russian Foundation for Basic Research (projects no. 16-01-00175).

\section{References}

1. Gromeka I. S. K teorii dvizheniia zhidkosti v uzkikh tsilindricheskikh trubakh [On the Theory of Fluid Motion in Narrow Cylindrical Tubes]. Collected works. Moscow, Publ. House of the Academy of Sciences of the USSR, 1952, pp. 149-171 (in Russian).

2. Zemlyanukhin A. I., Mogilevich L. I. Nonlinear Waves of Deformation in Cylindrical Shells. Izvestiya VUZ. Applied nonlinear dynamics, 1995, vol. 3, no. 1, pp. 52-58 (in Russian).

3. Erofeev V. I., Klyueva N. V. Solitons and nonlinear periodic strain waves in rods, plates, and shells (a review). Acoustical Physics, 2002, vol. 48, no. 6, pp. 643-655. DOI: 10.1134/1.1522030.

4. Zemlyanukhin A. I., Mogilevich L. I. Nonlinear waves in inhomogeneous cylindrical shells : a new evolution equation. Acoustical Physics, 2001, vol. 47, no. 3, pp. 303-307. DOI: 10.1134/1.1371586.

5. Arshinov G. A., Zemlyanukhin A. I., Mogilevich L. I. Two-dimensional solitary waves in a strained nonlinear viscoelastic medium. Acoustical Physics, 2000, vol. 46, no. 1, pp. 100-101.

6. Blinkova A. Iu., Ivanov S. V., Kovalev A. D., Mogilevich L. I. Mathematical and Computer Modeling of Nonlinear Waves Dynamics in a Physically Nonlinear Elastic Cylindrical Shells with Viscous Incompressible Liquid inside Them. Izv. Saratov Univ. (N. S.), Ser. Physics, 2012, vol. 12, iss. 2, pp. 12-18 (in Russian).

7. Blinkov Yu. A., Kovaleva I. A., Mogilevich L. I. Nonlinear Waves Dynamics Modeling in Coaxial Geometrically and Physically Nonlinear Shell Containing Viscous Incompressible Fluid in between. Bulletin of Peoples' Friendship University of Russia. Ser. Mathematics. Information Sciences. Physics, 2013, vol. 3, pp. 42-51 (in Russian).

8. Loitsianskii L. G. Mekhanika zhidkosti $i$ gaza
[And Fluid Mechanics Gas]. Moscow, Drofa, 2003, 840 p. (in Russian).

9. Vallander S. V. Lektsii po gidroaeromekhanike [Lectures on Hydromechanics]. Leningrad, Leningrad Univ. Press, 1978, 296 p. (in Russian).

10. Vol'mir A. S. Nelineinaia dinamika plastinok $i$ obolochek [Nonlinear Dynamics of Plates and Shells]. Moscow. Nauka, 1972, 432 p. (in Russian).

11. Vol'mir A. S. Obolochki v potoke zhidkosti i gaza: zadachi gidrouprugosti [Shell in the Liquid and Gas Flow : Tasks Hydroelasticity]. Moscow, Nauka, 1979, 320 p. (in Russian).

12. Schlichting H. Boundary Layer Theory. New York, USA, McCgraw-Hill, 1960, 605 p. (Russ. ed. : Schlichting H. Teoriia pogranichnogo sloia. Moscow, Nauka, 1974, 712 p.)

13. Chivilikhin S. A., Popov I. Yu., Gusarov V. V. Dynamics of nanotube twisting in a viscous fluid. Doklady Physics, 2007, vol. 52, no. 1, pp. 60-62.

14. Popov I. Yu., Rodygina O. A., Chivilikhin S. A., Gusarov V. V. Soliton in a nanotube wall and Stokes flow in the nanotube. Technical Physics Letters, 2010, vol. 36, no. 9, pp. 852-855. DOI: $10.1134 /$ S1063785010090221.

15. Gerdt V. P., Blinkov Yu. A. On Selection of Nonmultiplicative Prolongations in Computation of Janet Bases. Programming and Computer Software, 2007, vol. 33, no. 3, pp. 147-153. DOI: 10.1134/S0361768807030048.

16. Blinkov Yu. A., Gerdt V. P. Specialized Computer Algebra System Ginv. Programming and Computer Software, 2008, vol. 34, no. 2, pp. 67-80. DOI: 10.1007/s11086-008-2009-8.

17. Gerdt V. P., Blinkov Yu. A. Involution and difference schemes for the Navier-Stokes equations. Computer Algebra in Scientific Computing, vol. 5743 of Lecture Notes in Computer Science, 2009, pp. 94-105. DOI: 10.1007/978-3-642-041037_10. 